\title{
BMJ A nationwide Danish cohort study Open challenging the categorisation into right-sided and left-sided colon cancer
}

\author{
Per Jess, ${ }^{1,2}$ Iben Onsberg Hansen, ${ }^{2}$ Michael Gamborg, ${ }^{3}$ Tine Jess, ${ }^{4}$ on behalf of \\ the Danish Colorectal Cancer Group
}

To cite: Jess $P$, Hansen 10 , Gamborg M, et al.

A nationwide Danish cohort study challenging the categorisation into right-sided and left-sided colon cancer. BMJ Open 2013;3:e002608. doi:10.1136/bmjopen-2013002608

- Prepublication history and additional material for this paper are available online. To view these files please visit the journal online (http://dx.doi.org/10.1136/ bmjopen-2013-002608).

Received 17 January 2013 Revised 11 April 2013 Accepted 22 April 2013

This final article is available for use under the terms of the Creative Commons Attribution Non-Commercial 2.0 Licence; see http://bmjopen.bmj.com

${ }^{1}$ Department of Surgery, Roskilde Hospital, Roskilde, Denmark

${ }^{2}$ Faculty of Health Sciences, University of Copenhagen, Copenhagen, Denmark

${ }^{3}$ Institute of Preventive Medicine, Copenhagen University Hospital, Copenhagen, Denmark ${ }^{4}$ Department of Epidemiology Research, Statens Serum Institut, National Health Surveillance and Research, Copenhagen, Denmark

Correspondence to Dr Per Jess; pjss@regionsjaelland.dk

\section{ABSTRACT}

Objectives: The categorisation of colon cancer (CC) into right-sided (RCC) and left-sided (LCC) disease may not capture more subtle variances in aetiology and prognosis. In a nationwide study, we investigated differences in clinical characteristics and survival of RCC versus $L C C$ and of the complete range of $C C$ subsites.

Design: Prospective nationwide cohort study.

Setting: The database of the Danish Colorectal Cancer Group (DCCG).

Participants: 23487 CC patients.

Outcome measures: Overall survival (Kaplan-Meier plots) and mortality (HR from Cox proportional hazards regression analysis) according to $\mathrm{CC}$ localisation. For adjustment and stratification, we used age, sex, ASA score (the American Society of Anaesthesiologists score), tumour location and stage, number of lymph nodes harvested at operation, number of lymph nodes with metastases and presence of distant metastases.

Results: Patients with RCC had a higher median age at diagnosis (74.3 years) than patients with LCC (71.8 years; $p<0.0001)$. Overall, the proportion of patients who were women increased the closer the tumour site was to the small intestine. Although RCC patients had higher ASA scores than LCC patients $(p<0.0001)$, the highest ASA scores were observed in patients with cancer in the transverse and descending colon and at both colon flexures. While RCCs overall were more advanced than LCCs $(p<0.0001)$, the most advanced CCs were those of the descending colon, splenic flexure and caecum. RCC mortality was higher than LCC mortality only during the first 2 years (women: HR 1.13; $95 \% \mathrm{Cl} 1.06$ to 1.20 ; men: HR $1.27 ; 95 \% \mathrm{Cl} 1.20$ to 1.35 ), and relative to mortality from sigmoid CC, the highest mortality was observed from splenic flexure cancer ( $\mathrm{HR} 1.75 ; 95 \% \mathrm{Cl}$ 1.54 to 2.00 ).

Conclusions: The present data challenge the simple categorisation of CC into RCC and LCC.

\section{INTRODUCTION}

Colorectal cancer is one of the most commonly diagnosed cancers worldwide with

\section{ARTICLE SUMMARY}

Article focus

- Colon cancer (CC) is often classified into rightsided (RCC) and left-sided CC (LCC).

- It has been suggested that there are differences in epidemiology, pathology and prognosis between patients with cancer in the right and left sides of the colon due to different embryological development of the two segments of the colon which may result in different molecular biological patterns of the tumours.

- Recent studies have challenged this simple dichotomisation of CC into RCC and LCC.

\section{Key messages}

- Our nationwide cohort study of 23487 CC patients confirms that patients with RCC are older, more often females and have more advanced tumours and a worse prognosis than patients with left-sided cancer.

- However, a more detailed colon subsite analysis reveals a much more complex picture.

- The highest ASA scores are observed in patients with cancer of the transverse and descending colon and of both colon flexures. The most advanced CCs are those of the descending colon, splenic flexure and caecum. The highest mortality is seen in patients with splenic flexure cancer.

Strengths and limitations of this study

- The strength of the present study is the populationbased patient cohort with nearly complete (96-99\%) inclusion and follow-up of all Danish patients diagnosed with CC during the last 10 years.

- The study has potential limitations: Despite detailed information on patients, data on cancer recurrence have not been collected. Hence, we were only able to compare overall survival rather than cancer-free survival. However, this would not influence the already observed marked differences in clinical characteristics and survival by specific CC subsites. Further, we did not have genetic or biomarker information that could have served to further qualify the observations of these differences, and this merits further study. Finally, lack of information on chemotherapy was also a potential limitation. 
over 1.2 million new cancer cases and 608700 deaths estimated to occur annually. ${ }^{1}$

In 1990, Bufill was the first to propose that colon cancer (CC) located to the right (RCC) and left (LCC) sides of the colon, respectively, may rise from different biological pathways. ${ }^{2}$ Subsequently, it has been suggested that, in view of their being two different disease entities, RCC and LCC also differ in terms of patient characteristics, pathology and prognosis. ${ }^{3}$ A recent systematic review of the sparse literature on the topic supports the existence of such differences. ${ }^{4}$ The reason for this is uncertain, but a suggested explanation is the different embryological development of the two segments of the colon resulting in different molecular biological tumour patterns. ${ }^{5}{ }^{6}$ However, recent studies have challenged the simple dichotomisation of CC into RCG and LCC, since factors such as tumour stage, ${ }^{7}$ genetic alterations ${ }^{8}$ and molecular features ${ }^{9}$ vary markedly between colonic subsides irrespective of their localisation in the right or left sides of the colon. Such possible differences might have consequences for the planning of screening and for the treatment of patients with CC.

The aim of the present investigation was to examine the differences in patient characteristics, cancer pathology and survival according to RCG versus LCC localisation of CC and, according to more specific colon subsite localisation, using unique prospectively collected nationwide data from 2001 to 2010.

\section{MATERIALS AND METHODS}

On 1 May 2001, the Danish Colorectal Cancer Group (DCCG) established a nationwide database, ${ }^{10}$ and since then all patients with a first-time diagnosis of colorectal adenocarcinoma from all Danish surgical departments treating such patients have been prospectively registered in the database. Patients are identified by their Danish Civil Registration Number, a unique 10-digit personal number given to all Danish citizens at birth and ascribed to every Danish person since $1968 .^{11}$ The data reported to DCCG from the surgical departments in Denmark include demographics, clinical manifestation, tumour location, stage (UICC, Union for International Cancer Control), diagnostic and therapeutic procedures, and postoperative complications. Data on vital status are updated monthly through linkage to the Danish Civil Registration System. ${ }^{11}$ The completeness of data collection in DCCG is estimated annually and has increased from $96 \%$ to $99 \%$ in the period $2001-2010 .{ }^{10}$

The present study covers all Danish patients $(\mathrm{n}=23487)$ in DCCG with a diagnosis of CC from 1 January 2001 to 10 December 2010. From DCCG, we specifically obtained data on age, sex, ASA score (the American Society of Anaesthesiologists score), tumour location and stage (UICC), number of lymph nodes harvested at operation, number of lymph nodes with metastases and presence of distant metastases.
Localisation of CC was treated both according to the specific subsites and according to the suggested categorisation into RCC ( $\mathrm{n}=10 \mathrm{855}$; caecum, ascending colon, hepatic flexure or transverse colon) and LCC ( $\mathrm{n}=12$ 632; from the splenic flexure to the sigmoid colon, both included; the border between the sigmoid colon and rectum was defined as $15 \mathrm{~cm}$ from the anal verge).

\section{Statistical analyses}

Non-parametric statistics (median, range, $\chi^{2}$ test, and Mann-Whitney test) were used to describe the demographic, clinical and pathological characteristics of the patient population. $p<0.05$ were considered to be statistically significant. Patients were followed from the date of CC diagnosis to emigration, death or end of the study (13 October 2011), whichever occurred first. Overall survival among patients with RCG vs LCG was illustrated by Kaplan-Meier plots for women and men, respectively, and was compared by log-rank test. Mortality from any course from RCC relative to mortality from LCC was estimated by HRs obtained by performing Cox proportional hazard regression analysis with time since diagnosis as the underling time axis, stratified on gender and adjusted for age. Next, the impact of the ASA score, number of lymph nodes harvested at operation, number of lymph nodes with metastases, presence of distant metastases and UICC stage on the relative difference in mortality from RCC and LCC was investigated by performing adjusted analyses. Finally, analyses were performed to compare the survival among patients with cancer of the caecum, ascending colon, hepatic flexure, transverse colon, splenic flexure and descending colon, respectively, with the survival among patients with sigmoid CC. All analyses were performed using STATAV.9.2.

\section{RESULTS}

\section{Age, gender and ASA score}

The median age at diagnosis of RCG was 74.3 years (range 18.5-104.8 years) vs 71.8 years (range 13.2-100.9) for LCC $(p<0.0001)$. The same pattern was seen when examining CC subsites (table 1 ).

A significantly higher proportion of RCC (56.8\%) than LCC $(46 \%)$ patients were women $(p<0.0001)$. Likewise, when examining CC subsites, the proportion of patients who were women increased the closer the tumour site was to the small intestine. However, among patients with hepatic flexure cancers, women were not in excess (table 1 ).

A modestly higher ASA score was observed in RCC patients as compared to LCC patients $(\mathrm{p}<0.0001)$, with $30.6 \%$ of RCC patients having ASA scores of $\geq 3$ compared to $27.9 \%$ of LCC patients. However, focusing on CG subsites, ASA scores of $\geq 3$ were most common in patients with CG located to the hepatic flexure, the transverse colon as well as the splenic flexure and the descending colon (table 1). 


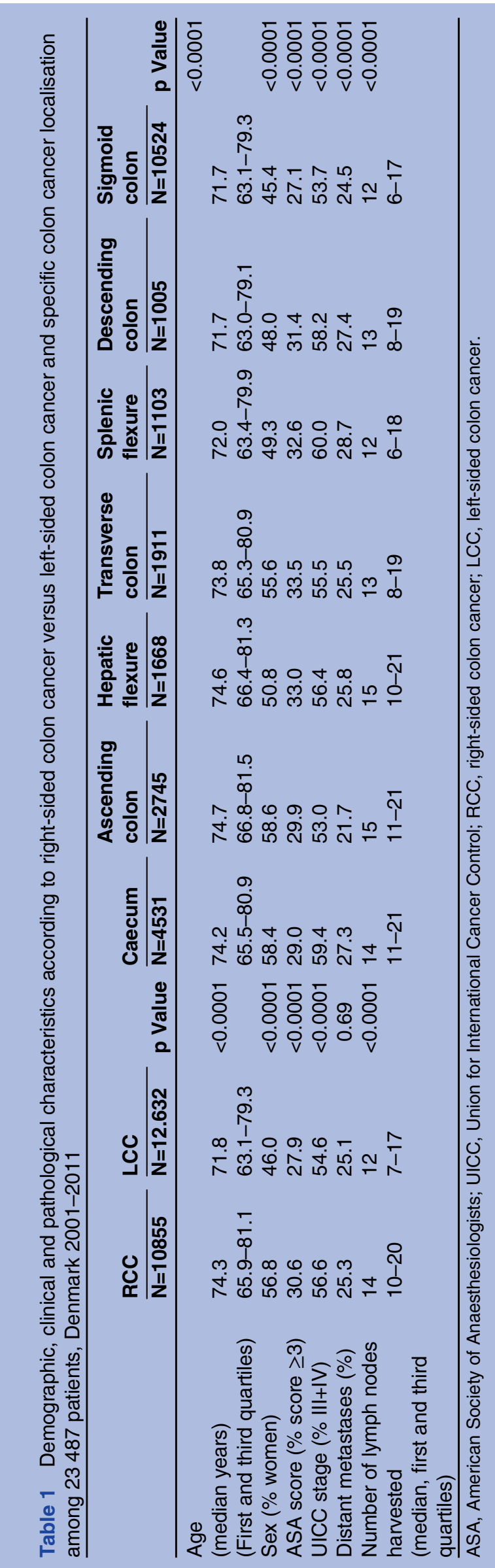

Pathology

Although RCC patients as a group had significantly more advanced UICG stage than patients with LCC $(\mathrm{p}=0.003), \mathrm{CC}$ subsite analyses revealed that caecal, splenic flexure and descending CCs were in the most advanced stages (table 1 ).

The median number of lymph nodes harvested was slightly higher in patients with RCC $(\mathrm{n}=14)$ than in patients with LCC $(\mathrm{n}=12) \quad(\mathrm{p}<0.0001)$, which corresponded to the observation for CC subsites. However, a similar proportion of RCC $(25.3 \%)$ and LCC $(25.1 \%)$ patients was found to have distant metastases $(p=0.69)$ and CC subsite analysis showed that the highest proportion of distant metastases was observed in patients with $\mathrm{CC}$ of the caecum, splenic flexure or descending colon (table 1).

\section{Survival}

Patients were followed for up to 10.8 years (median 26 months) from diagnosis of CC. Kaplan-Meier plots of 5 -year overall survival in women and men (figure 1) showed overall survival to be poorer in patients with RCC than in patients with LCC $(p<0.0001)$. This was in age-adjusted Cox regression analyses found to be explained by excess mortality in patients with RCC as compared to patients with LCC within the first 2 years after diagnosis $\left(\mathrm{HR}_{\text {women }} 1.13, \mathrm{p}<0.0001 ; \mathrm{HR}_{\text {men }} 1.27\right.$, $\mathrm{p}<0.0001$; table 2). In the following $3-5$ years after CC diagnosis, mortality tended to be lower in patients with RCC than in patients with LCG $\left(\mathrm{HR}_{\text {women }} 0.87, \mathrm{p}=0.02\right.$; $\mathrm{HR}_{\text {men }}$ 0.95, $\mathrm{p}=0.31$; table 2).

Adjustment for ASA score did not change the presented estimates (table 2). Adjustment for the number of removed lymph nodes resulted in a stronger association between localisation and mortality (especially in the first 2 years; table 2), which was found to be explained by a positive association between RCC and the

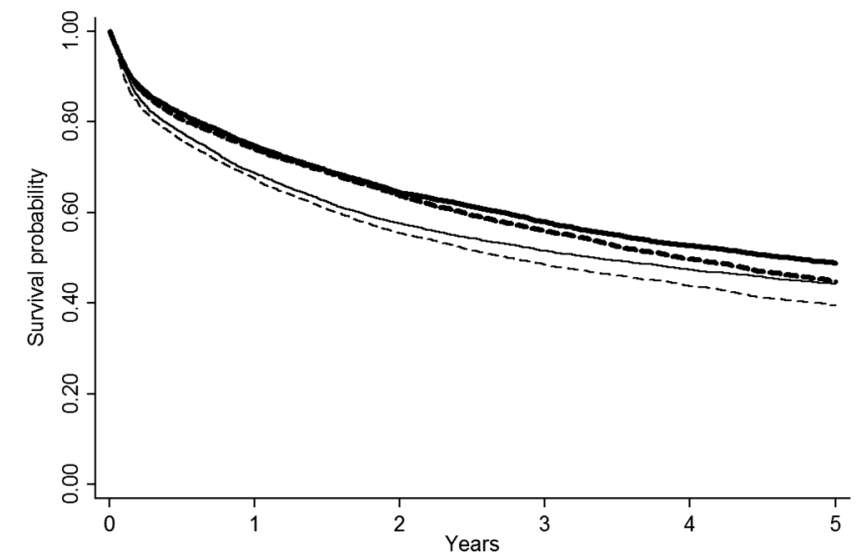

Figure 1 Kaplan-Meier plot of survival according to time since diagnosis of right-sided (thin lines) and left-sided (black lines) colon cancer in 11977 Danish women (2001-2011) (solid lines) and 11510 Danish men (2001-2011) (dashed lines). 
Table 2 HRs for mortality from right-sided colon cancer relative to mortality from left-sided colon cancer

\begin{tabular}{|c|c|c|c|c|}
\hline & $\begin{array}{l}\mathbf{N} \\
\text { Cases/no. of patients }\end{array}$ & $\begin{array}{l}\text { Women } \\
\text { HR }(95 \% \mathrm{Cl}) \\
\end{array}$ & $\begin{array}{l}\mathbf{N} \\
\text { Cases/no. of patients }\end{array}$ & $\begin{array}{l}\text { Men } \\
\text { HR }(95 \% \mathrm{Cl}) \\
\end{array}$ \\
\hline \multicolumn{5}{|l|}{ Overall } \\
\hline Years 1-2 & $4579 / 11963$ & 1.13 (1.06 to 1.20$)$ & $4457 / 11515$ & $1.27(1.20$ to 1.35$)$ \\
\hline Years 3-5 & $1237 / 6270$ & 0.87 (0.77 to 0.97$)$ & $1437 / 5960$ & $0.95(0.85$ to 1.05$)$ \\
\hline \multicolumn{5}{|l|}{ Adjusted for ASA score } \\
\hline Years 1-2 & $3924 / 10787$ & 1.15 (1.08 to 1.23$)$ & $3852 / 10439$ & $1.27(1.19$ to 1.35$)$ \\
\hline Years 3-5 & $1127 / 5811$ & 0.86 (0.76 to 0.97$)$ & $1318 / 5550$ & $0.94(0.84$ to 1.05$)$ \\
\hline \multicolumn{5}{|l|}{$\begin{array}{l}\text { Adjusted for no. of harvested } \\
\text { lymph nodes }\end{array}$} \\
\hline Years 1-2 & $3280 / 10192$ & $1.42(1.32$ to 1.52$)$ & $3152 / 9703$ & $1.57(1.47$ to 1.69$)$ \\
\hline Years 3-5 & $1121 / 5889$ & 0.91 (0.81 to 1.03$)$ & $1296 / 5562$ & $1.02(0.91$ to 1.15$)$ \\
\hline \multicolumn{5}{|l|}{$\begin{array}{l}\text { Adjusted for no. of lymph } \\
\text { nodes with metastases }\end{array}$} \\
\hline Years 1-2 & 2913/9741 & 1.16 (1.07 to 1.25$)$ & $2782 / 9253$ & $1.30(1.20$ to 1.40$)$ \\
\hline Years 3-5 & $1103 / 5805$ & 0.86 (0.76 to 0.97$)$ & $1275 / 5490$ & 0.98 (0.88 to 1.10$)$ \\
\hline \multicolumn{5}{|l|}{$\begin{array}{l}\text { Adjusted for presence of } \\
\text { distant metastases }\end{array}$} \\
\hline Years 1-2 & $4365 / 11591$ & $1.22(1.15$ to 1.29$)$ & $4293 / 11195$ & $1.35(1.27$ to 1.43$)$ \\
\hline Years 3-5 & $1193 / 6087$ & $0.92(0.82$ to 1.04$)$ & $1402 / 5813$ & $1.03(0.92$ to 1.14$)$ \\
\hline \multicolumn{5}{|l|}{ Adjusted for UICC stage } \\
\hline Years 1-2 & $4176 / 11296$ & $1.18(1.10$ to 1.25$)$ & $4137 / 10917$ & $1.31(1.24$ to 1.40$)$ \\
\hline Years 3-5 & $1185 / 6043$ & 0.87 (0.78 to 0.99$)$ & $1377 / 5736$ & $0.99(0.89$ to 1.11$)$ \\
\hline \multirow[t]{2}{*}{ Global model } & $2649 / 9106$ & 1.28 (1.18 to 1.39$)$ & $2549 / 8674$ & $1.36(1.25$ to 1.47$)$ \\
\hline & $1036 / 5473$ & 0.93 (0.82 to 1.06$)$ & $1194 / 5175$ & 1.06 (0.94 to 1.19$)$ \\
\hline \multirow{2}{*}{$\begin{array}{l}\text { Unadjusted on data with } \\
\text { complete conf. info. }\end{array}$} & 2649/9106 & $1.29(1.19$ to 1.40$)$ & $2549 / 8674$ & $1.34(1.24$ to 1.45$)$ \\
\hline & $1036 / 5473$ & 0.87 (0.77 to 0.98$)$ & $1194 / 5175$ & 0.97 (0.86 to 1.09$)$ \\
\hline
\end{tabular}

number of lymph nodes removed and an inverse association between the number of lymph nodes removed and mortality.

Adjustment for the number of lymph nodes with metastases had little impact on the difference in mortality from RCC relative to LCC (table 2). However, further analyses revealed an interaction between $\mathrm{CC}$ localisation and the number of lymph nodes with metastases, which was most pronounced in women $(\mathrm{p}<0.001)$, and the excess 1-2 year mortality from RCC relative to LCC among female patients was only present among women with one or more lymph nodes with metastases, whereas the mortality was decreased in RCG women as compared to LCG women without lymph node metastases (table 3).

The presence of distant metastases and the UICC stage did not explain the difference in mortality between RCC and LCC (table 2).

In a sensitivity analysis of survival among a subset of the population (9106 women and 8674 men) with information on all potential confounders (the ASA score, number of lymph nodes harvested, number of lymph nodes with metastases, presence of distant metastases,

Table 3 HRs for mortality from right-sided colon cancer relative to mortality from left-sided colon cancer according to number of lymph nodes with metastases

\begin{tabular}{|c|c|c|c|c|}
\hline & \multicolumn{2}{|l|}{ Women } & \multicolumn{2}{|l|}{ Men } \\
\hline & $\begin{array}{l}\text { Years 1-2 } \\
\text { HR }(95 \% \mathrm{Cl})\end{array}$ & $\begin{array}{l}\text { Years 3-5 } \\
\text { HR }(95 \% \text { Cl9 }\end{array}$ & $\begin{array}{l}\text { Years 1-2 } \\
\text { HR }(95 \% \mathrm{Cl})\end{array}$ & $\begin{array}{l}\text { Years 3-5 } \\
\text { HR }(95 \% \mathrm{Cl})\end{array}$ \\
\hline \multicolumn{5}{|c|}{ Lymph nodes with metastases } \\
\hline 0 & $0.83(0.74$ to 0.94$)$ & $0.75(0.63$ to 0.89$)$ & $1.17(1.04$ to 1.31$)$ & 1.04 (0.89 to 1.22$)$ \\
\hline $1-3$ & 1.33 (1.15 to 1.53$)$ & 0.87 (0.69 to 1.10$)$ & $1.27(1.10$ to 1.48$)$ & 0.98 (0.78 to 1.22$)$ \\
\hline$>4$ & 1.55 (1.36 to 1.77$)$ & $1.22(0.94$ to 1.58$)$ & $1.54(1.35$ to 1.75$)$ & 0.86 (0.68 to 1.11$)$ \\
\hline
\end{tabular}

Cox proportional hazard models of mortality from right-sided vs left-sided colon cancer in patients with0, 1-3 and >4 lymph nodes with metastases, respectively. Analyses were adjusted for age. 
and UICC stage), we found the 1-2 year HR for women to be 1.29 (95\% CI 1.19 to 1.40) in a crude model and 1.28 (95\% CI 1.18 to 1.39 ) in a full model including all these variables. In men, the crude $1-2$ year HR was 1.34 (95\% CI 1.24 to 1.45 ) and the fully adjusted HR was 1.36 (95\% CI 1.25 to 1.47 ). The $3-5$ year HR was 0.87 (95\% CI 0.77 to 0.97 ) for women and 0.97 (95\% CI 0.86 to 1.09) for men in crude analysis, whereas the fully adjusted 3-5 year HR was 0.93 (95\% CI 0.82 to 1.06 ) for women and 1.06 (95\% CI 0.94 to 1.19) for men.

Analyses were repeated for CC subsites using sigmoid colon localisation as the reference (table 4). Relative mortality was higher for all other CC subsites than sigmoid CC both in women and men during the first 2 years after CC diagnosis (table 4 ). Interestingly, mortality from cancer of the caecum and ascending colon were closest to mortality from sigmoid cancer (table 4), whereas mortality was $28-50 \%$ increased for cancer of the hepatic flexure, $45-55 \%$ increased for cancer of the transverse colon, and, notably, $71-75 \%$ increased for cancer of the splenic flexure relative to mortality from sigmoid cancer (table 4). During years 3-5 following cancer diagnosis, mortality for the investigated CC subsites were not different from mortality from sigmoid CC except for a significantly lower relative mortality from cancer of the ascending colon (table 4). Adjustment for the ASA score, number of harvested lymph nodes, number of lymph nodes with metastases, presence of distant metastases and UICC stage had a minor impact on these observations (data not shown).

\section{DISCUSSION}

Our nationwide cohort study of 23487 prospectively followed CG patients revealed that although patients with RCC were, overall, older, more often of female gender, had modestly higher ASA scores and more advanced UICC stages than patients with LCC, the picture was indeed more complex when assessing the underlying CC subsites. Mortality from RCG was only relatively higher than mortality from LCC during the first 2 years after CC diagnosis, probably due to the observation that the majority of recurrences in colorectal cancer occurs within the first 2 years after primary treatment. ${ }^{12}$ In analyses of CC subsites, the highest relative mortality was from cancer of the splenic flexure (ie, belonging to the LCC category), while the lowest relative mortality was from sigmoid cancer (also belonging to the LCC category). The differences in mortality from CC of different colon subsites was only to a minor extent explained by the ASA scores, number of harvested lymph nodes, number of lymph nodes with metastases, presence of distant metastases and UICC stage. Overall, our observations challenge the simple categorisation of CC into RCC and LCC.

The primary strength of our study was the populationbased patient cohort with nearly complete (96-99\%) inclusion and follow-up of all Danish patients diagnosed

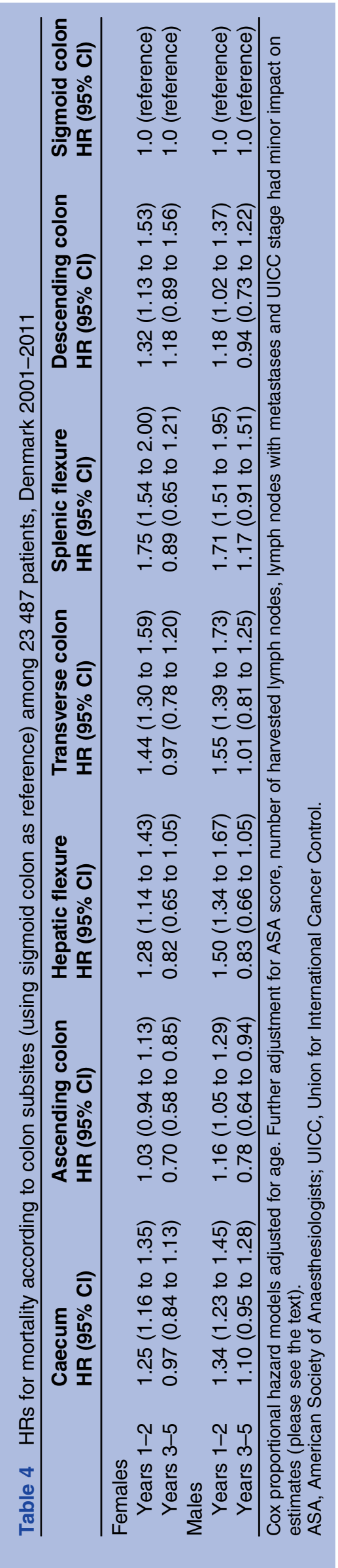


with CC during the last 10 years. Using the 10-digit personal identification number given to all Danish citizens at birth, complete information on vital status could be obtained from the Danish Central Person Registry. Further, detailed information on the patient characteristics, surgical procedures including harvesting of lymph nodes, lymph node and tumour pathology, and UICC stages were available.

The study also had potential limitations to consider. Despite detailed information on patients, data on cancer recurrence had not been collected. Hence, we were only able to compare overall survival rather than cancer-free survival between RCC and LCC patients or by CC subsite. Lack of info on chemotherapy is also a potential limitation of the study, since chemotherapy may influence survival. However, the highest frequency of stage III and IV cancers, normally leading to chemotherapy, was seen in splenic flexure cancers, and these cancers still had the highest relative mortality compared to other sites. It may also be seen as a limitation of the study that patients with synchronous or overlapping site CCs have been categorised according to the localisation of the cancer with the highest UICC stage. However, only approximately $5 \%$ of CCs are synchronous. ${ }^{13}$ Potentially, we may oversee mild LCCs in patients with higher stage synchronous RCCs (or vice versa), but this is judged to have little impact on results and interpretation of these. Further, we did not have genetic or biomarker information that could have served to further qualify the observations of the differences between the groups. This merits further study. Lastly, we did not have information on all potential confounders on all individuals. We handled this by first running analysis adjusting for one confounder per se (with little impact of most variables) and secondly running sensitivity analyses using the select subset of patients with information on all potential confounders. This resulted in removal of approximately one-third of patients, which in turn appeared to influence the overall crude HR markedly more than did the following adjustment for all potential confounders, hence suggesting selection bias in this subset of patients to be a greater problem than confounding by the mentioned factors.

In the present study, we observed an almost linear relationship between tumour location and age; that is, the more proximal the tumour was located, the higher was the median age among patients. This did obviously result in a higher median age among RCC patients than among LCC patients. However, the same would have been the case had the division between groups been made at any other place along the colon (comparing proximal cancers with distal cancers), and it may not necessarily support the theory of RCC and LCC being two separate disease entities. The observed age distribution may be explained by the diagnostic delay of cancers of the right side of the colon due to weaker symptoms. The sojourn time of preclinical colorectal cancer is estimated to range from 4.5 to 5.8 years $^{14}$ and the difference in median age of patients with cancer in the caecum and cancer in the sigmoid colon was only 2.5 years, hence likely reflecting the diagnostic delay. Another part of the explanation could be that the diagnostic accuracy of colonoscopy is lower in the right side than in the left side of the colon ${ }^{15}$ and lower in women, which would further explain the observed gender differences between patients with RCC and LCC. ${ }^{15}$

In accordance with a few earlier studies, ${ }^{3}{ }^{16}$ we observed higher ASA scores among RCC patients than LCC patients. However, more detailed analyses of CC subsites revealed that ASA scores $\geq 3$ were most often seen in patients with cancer of the hepatic flexure, transverse colon as well as of the splenic flexure and descending colon, that is, cancers belonging to both the RCC and LCC categories, hence again questioning the simple dichotomisation of CG.

We observed patients with RCC to have significantly more advanced cancer stages than patients with LCC in accordance with a previous systematic literature review. ${ }^{4}$ However, CG subsite analyses again revealed a more complex picture with the most advanced stages and distant metastases observed for cancers of the caecum, splenic flexure and descending colon. This is in accordance with the study of Benedix $e t a l^{7}$, who, in an analysis of 29568 CC patients, found the highest rate of prognostically favourable UICC stages (I or II) among carcinomas of the descending colon (55.3\%) and ascending colon $(53.6 \%)$, whereas the lowest rates were found for the splenic flexure $(49.0 \%)$ and the caecum $(47.7 \%)$. Further, cancers of the caecum and splenic flexure (ie, representing both RCC and LCC) were most advanced with regard to the metastatic spread (UICC stage IV).

In the present study, overall survival was poorer in patients with RCC than in patients with LCC, in accordance with the previous systematic review. ${ }^{4}$ However, the review also revealed that when eliminating confounding by differences in patient or tumour characteristics, the prognostic picture became more complex in most studies. Suttie et $a l^{17}$ found that age, tumour stage and acute presentation had a significant impact on differences in survival between patients with RCC and LCC, in accordance with Faivre-Finn $e t a l^{18}$ who also found tumour location per se to associate with survival. This has, however, been questioned by others. ${ }^{3}{ }^{16} \mathrm{We}$ observed mortality from RCC (relative to LCC) to be increased only during the first 2 years after CC diagnosis and to be most pronounced in men. The ASA score, UICC stage and presence of distant metastases had little impact on the difference in mortality from RCC and LCC, whereas an interaction with the number of lymph nodes with metastases was observed. However, when assessing CC subsites and using sigmoid colon localisation as reference, the highest relative mortality was observed in patients with splenic flexure cancer. This is in accordance with one study ${ }^{19}$ but in contrast to another previous study, ${ }^{20}$ and again challenges the concept of RCC and LCC as two distinct disease entities. 
It remains unknown why patient characteristics, cancer pathology and survival differ between CC subsites. ${ }^{8}$ However, it has recently been shown that biological features, such as the rate of cancer gene mutations in the form of microsatellite instability (MSI), K-RAS and BRAF mutations, differ along colonic subsites ${ }^{8}$ and therefore do not fit the theory of CC simply arising from the embryological right or left side of the colon. ${ }^{21}$ Yamauchi et $a l^{9} 22$ found that the frequencies of MSI-high, CpG island methylator phenotype (CIMP)-high and BRAF mutations increased in a statistically linear fashion from the rectum to the ascending colon with caecal cancer appearing to represent a unique subtype, characterised by a high frequency of KRAS mutations. The findings indicate that the molecular pathological changes evolve gradually as a continuum through bowel subsites rather than abruptly at the splenic flexure. However, Benedix et $a l^{8}$ found that MSI status, KRAS and BRAF mutation rates varied remarkably among the colonic subsites irrespective of a right-sided or left-sided origin and without gradually evolving through the colon. The last observations are somewhat in accordance with the results of the present study, but the few and conflicting data on the subject underscore the need for further studies of colorectal neoplasia by CC subsite at the molecular, pathological and epidemiological levels. ${ }^{23} 24$

In conclusion, the present nationwide populationbased cohort study of more than 20000 prospectively followed CC patients revealed that,overall,patients with RCC may differ from patients with LCC in terms of age, gender, ASA scores, cancer stage and survival. However, these findings seem to cover a much more complex pattern of disease, especially with cancer of the splenic flexure showing poor stage and prognosis. Our study strongly challenges the simple classification of CC into right-sided versus left-sided disease and underscores the need for a more subtle CC classification, in accordance with recent molecular biological findings. A better classification will enhance our possibilities to provide optimal surveillance and treatment of CC.

Contributors The study was conceived and designed by PJ, IOH, MG and TJ. Statistical analyses were performed by MG. All authors were involved in the interpretation of data. PJ drafted the manuscript, which was critically revised by all authors, who also approved the final version of the manuscript.

Funding This research received no specific grant from any funding agency in the public, commercial or not-for-profit sectors.

Competing interests None.

Ethics approval The Danish National Committee on Biomedical Research Ethics and the Danish Data Protection Agency have approved the use of the DCCG database for scientific purposes and also for the present study.

Provenance and peer review Not commissioned; externally peer reviewed.

Data sharing statement No additional data are available.

\section{REFERENCES}

1. Jemal A, Bray F, Center MM, et al. Clobal Cancer Statistics. $C A$ Cancer J Clin 2011;61:69-90.

2. Bufill JA. Colorectal cancer: Evidence for distinct genetic categories based on proximal or distal location. Ann Int Med 1990;113:779-88.

3. Benedix F, Kube R, Meyer F, et al. Comparison of 17641 patients with right- and left-sided colon cancer: differences in epidemiology, perioperative course, histology, and survival. Dis Colon Rectum 2010;53:57-64.

4. Hansen IO, Jess P. Possible better long-term survival in left versus right-sided colon cancer-a systematic review. Dan Med J 2012;59: A4444.

5. Elnatan J, Gosh HS, Smith DR. C-KI-RAS activation and the biological behaviour of proximal and distal colonic adenocarcinomas. Eur J Cancer 1996;32A:491-7.

6. Hutchins G, Southward K, Handley K, et al. Value of mismatch repair, KRAS, and BRAF mutations in predicting recurrence and benefits from chemotherapy in colorectal cancer. $J$ Clin Oncol 2011;29:1261-70.

7. Benedix F, Schmidt U, Mroczkowski $P$, et al. Colon carcinomaClassification into right and left sided cancer or according to colonic subsites?-Analysis of 29568 patients. EJSO 2011;37:134-9.

8. Benedix F, Meyer F, Kube R, et al. Influence of anatomical subsite on the incidence of microsatellite instability, and KRAS and BRAF mutation rates in patients with colon carcinoma. Pathol Res Pract 2012;208:592-7.

9. Yamauchi M, Morikawa T, Kuchiba A, et al. Assessment of colorectal cancer molecular features along bowel subsites challenges the conception of distinct dichotomy of proximal versus distal colorectum. Gut 2012;61:847-54.

10. Danish Colorectal Cancer Group (DCCG). 2012. http://www.DCCG.dk

11. Pedersen CB, Gotzsche H, Moller JO, et al. The Danish National Civil Registration System. A cohort of eight million persons. Dan Med Bull 2006;53:441-9.

12. Anthony T, Fleming JB, Bieligk SC, et al. Postoperative colorectal cancer surveillance. J Am Coll Surg 2000;190:737-49.

13. Arenas RB, Fichera A, Mhoon D, et al. Incidence and therapeutic implications of synchronous colonic pathology in colorectal adenocarcinoma. Surgery 1997;122:706-10.

14. Brenner $\mathrm{H}$, Altenhofen L, Katalinic $\mathrm{A}$, et al. Sojourn time of preclinical colorectal cancer by sex and age: estimates from the German National Screening Colonoscopy Database. Am J Epidemiol 2011;174:1140-6.

15. Singh H, Nugent Z, Demers AA, et al. Rate and predictors of early/ missed colorectal cancers after colonoscopy in Manitoba: a population-based study. Am J Gastroenetrol 2010;105:2588-96.

16. Weiss JM, Pfau PR, O'Connor ES, et al. Mortality by stage for rightversus left-sided colon cancer: analysis of surveillance, epidemiology, and end results-Medicare data. J Clin Oncol 2011;29:4401-9.

17. Suttie SA, Shaikh I, Mullen R, et al. Outcome of right- and left-sided colonic and rectal cancer following surgical resection. Colorectal Dis 2011;13:884-9.

18. Faivre-Finn C, Bouvier-Benhamiche AM, Phelip JM, et al. Colon cancer in France: evidence for improvement in management and survival. Gut 2002;51:60-4.

19. Gatta G, Ciccolallo L, Capocaccia R, et al. Differences in colorectal cancer survival between European and US populations: the importance of sub-site and morphology. Eur J Cancer 2003;39:2214-22.

20. Nakogoe T, Sawai T, Tsuji T, et al. Carcinoma of the splenic flexure: multivariate analysis of predictive factors for clinicopathological characteristics and outcome after surgery. $J$ Gastroenterol 2000;35:528-35.

21. Minoo P, Zlobec I, Peterson M, et al. Characterization of rectal, proximal and distal colon cancers based on clinicophatological, molecular and protein profiles. Int J Oncol 2010;37:707-18.

22. Yamauchi M, Lochhead P, Morikawa T, et al. Colorectal cancer: a tale of two sides or a continuum? Gut 2012;61:794-7.

23. Prichard CC, Grady WM. Colorectal cancer molecular biology moves into clinical practice. Gut 2011;60:116-29.

24. Ogino S, Chan AT, Fuchs CS. Molecular pathological epidemiology of colorectal neoplasia: an emerging transdisciplinary and interdisciplinary filed. Gut 60:397-411. 